\section{Artificial neural network accurately predicts mortality from end-stage liver disease}

The shortage of liver donors, and length of transplantation waiting lists, confer considerable importance on determining the prognosis of patients with chronic liver disease so that allocation of donor organs can be prioritized. The Model for End-stage Liver Disease (MELD) score has been adopted for this purpose, although it frequently fails to predict mortality accurately, possibly because of complex relationships between the biological variables it evaluates.

Cucchetti et al. compared the ability of an artificial neural network (ANN) with that of the MELD score to predict mortality in patients with end-stage liver disease who were on waiting lists for liver transplantation. The ANN was trained to predict 3-month mortality using clinical data from 188 patients, and then tested on data from an internal validation group of 63 patients and an external cohort of 137 patients. The mortality predictions of the ANN were compared with those of the MELD score by looking at the areas under receiveroperating characteristic curves (AUC); the ANN mortality predictions were significantly more accurate than the MELD predictions for both the internal validation group (AUCs 0.95 vs $0.85 ; P=0.032$ ) and the external cohort (AUCs 0.96 vs $0.86 ; P=0.044$ ).

The ANN was considered to be superior to the MELD score for prioritizing liver transplant candidates, and the authors suggest that it has potential as a reliable tool for reducing mortality among patients awaiting transplantation.

Original article Cucchetti A et al. (2007) Artificial neural network is superior to MELD in predicting mortality of patients with end-stage liver disease. Gut 56: $253-258$

\section{Superior outcomes for surgical pancreatic duct decompression}

Patients with chronic pancreatitis and a dilated pancreatic duct are recommended to undergo ductal decompression. Cahen and colleagues compared endoscopic and surgical drainage of the pancreatic duct in their prospective, randomized study.
In total, 39 patients were randomly allocated to undergo either endoscopic transampullary drainage $(n=19)$ or surgical pancreaticojejunostomy $(n=20)$. Baseline assessments included standard laboratory and symptom evaluations, quality-of-life scores (measured using the 36-item Short-Form General Health Survey), and Izbicki pain score (a pancreatitisspecific pain questionnaire). These assessments were repeated at week 6 and months 3 , $6,12,18$ and 24 after the initial procedure.

The maximum Izbicki pain score is 100 ; after adjustment for baseline scores, the mean Izbicki pain score over the 2 years of follow-up was 24 points lower in surgically treated than in endoscopically treated patients $(95 \% \mathrm{Cl} 11-$ 36, $P<0.001$ ). Complete or partial pain relief was achieved in significantly more surgically treated than endoscopically treated patients ( $75 \%$ vs $32 \%, P=0.007$ ). Short-Form 36 physical health scores were markedly lower in the endoscopy group than in the surgery group, and endoscopically treated patients underwent more interventions overall (median eight vs three procedures, $P<0.001$ ).

The authors conclude that surgical treatment has better 2-year outcomes than endoscopic treatment, although their results might not be applicable to all patients with chronic pancreatitis; patients in this study had complex pathology, and those with inflammatory masses were excluded. In patients with lessextensive disease, endoscopic treatment might still be valuable.

Original article Cahen DL et al. (2007) Endoscopic versus surgical drainage of the pancreatic duct in chronic pancreatitis. N Engl J Med 356: 676-684

\section{Association between humans and $\boldsymbol{H}$. pylori originated in Africa}

The point in time at which Helicobacter pylori became associated with humans remains controversial; however, the variation reported among $H$. pylori strains suggests that the phylogeny of these bacteria reflects human geographical migrations throughout history.

An international team of scientists used genetic sequence data from $769 \mathrm{H}$. pylori isolates to determine whether patterns in their geographic distribution mimic human migration patterns. Assignment of $H$. pylori isolates to geographically distinct populations revealed 